\title{
Periodic solutions to a generalized Liénard neutral functional differential system with $p$-Laplacian
}

\section{Qing Yang and Bo Du*}

\section{"Correspondence:}

dubo7307@163.com

Department of Mathematics,

Huaiyin Normal University, Huaiyin,

Jiangsu 223300, P.R. China

\begin{abstract}
By means of the generalized Mawhin's continuation theorem, we present some sufficient conditions which guarantee the existence of at least one $T$-periodic solution for a generalized Liénard neutral functional differential system with $p$-Laplacian.

MSC: 34B15; 34L30
\end{abstract}

Keywords: periodic solutions; neutral equations; generalized Mawhin's continuation theorem

\section{Introduction}

This paper is devoted to investigating the following $p$-Laplacian Liénard neutral differential system:

$$
\left(\varphi_{p}\left((x(t)-B x(t-\tau))^{\prime}\right)\right)^{\prime}+f(x(t)) x^{\prime}(t)+g(x(t-\gamma(t)))=e(t),
$$

where $x(t)=\left(x_{1}(t), x_{2}(t), \ldots, x_{n}(t)\right)^{\top}$;

$$
\begin{aligned}
& \varphi_{p}: \mathbb{R}^{n} \rightarrow \mathbb{R}^{n}, \quad \varphi_{p}(x)=|x|^{p-2} x=\left(\sqrt{\sum_{i=1}^{n} x_{i}^{2}}\right)^{p-2} x, \quad p>1 ; \\
& f(x)=\left(f_{1}\left(x_{1}\right), f_{2}\left(x_{2}\right), \ldots, f_{n}\left(x_{n}\right)\right), \quad f_{i}\left(x_{i}\right) \in C(\mathbb{R}, \mathbb{R}), i=1,2, \ldots, n ; \\
& g(x)=\left(g_{1}\left(x_{1}\right), g_{2}\left(x_{2}\right), \ldots, g_{n}\left(x_{n}\right)\right)^{\top}, \quad g_{i}\left(x_{i}\right) \in C(\mathbb{R}, \mathbb{R}), i=1,2, \ldots, n ;
\end{aligned}
$$

$e \in C\left(\mathbb{R}, \mathbb{R}^{n}\right)$ with $e(t+T)=e(t) ; \gamma \in C(\mathbb{R}, \mathbb{R})$ with $\gamma(t+T)=\gamma(t) ; \tau$ is a given constant; $B=\left[b_{i j}\right]_{n \times n}$ is a real matrix with $|B|=\left(\sum_{i=1}^{n} \sum_{j=1}^{n}\left|b_{i j}\right|^{2}\right)^{1 / 2}$.

When the matrix $B$ is a constant, Zhang [1] studied the properties of a difference operator $A$ and obtained the following results: define the operator $A$ on $C_{T}$

$$
A: C_{T} \rightarrow C_{T}, \quad[A x](t)=x(t)-c x(t-\tau), \quad \forall t \in \mathbb{R},
$$

where $C_{T}=\{x: x \in C(\mathbb{R}, \mathbb{R}), x(t+T) \equiv x(t)\}, c$ is a constant. If $|c| \neq 1$, then $A$ has a unique continuous bounded inverse $A^{-1}$ satisfying

$$
\left[A^{-1} f\right](t)= \begin{cases}\sum_{j \geq 0} c^{j} f(t-j \tau), & \text { if }|c|<1, \forall f \in C_{T}, \\ -\sum_{j \geq 1} c^{-j} f(t+j \tau), & \text { if }|c|>1, \forall f \in C_{T} .\end{cases}
$$

○ 2012 Yang and Du; licensee Springer. This is an Open Access article distributed under the terms of the Creative Commons Attribution License (http://creativecommons.org/licenses/by/2.0), which permits unrestricted use, distribution, and reproduction in any medium, provided the original work is properly cited. 
On the basis of Zhang's work, $\mathrm{Lu}$ [2] further studied the properties of the difference operator $A$ and gave the following inequality properties for $A$ :

(1) $\left\|A^{-1}\right\| \leq \frac{1}{|1-| k||}$;

(2) $\int_{0}^{T}\left|\left[A^{-1} f\right](t)\right| d t \leq \frac{1}{|1-| k \mid} \int_{0}^{T}|f(t)| d t, \forall f \in C_{T}$;

(3) $\int_{0}^{T}\left|\left[A^{-1} f\right](t)\right|^{2} d t \leq \frac{1}{|1-| k||} \int_{0}^{T}|f(t)|^{2} d t, \forall f \in C_{T}$.

After that, by using the above results, many researchers studied the existence of periodic solutions for some kinds of differential equations; see [3-7]. In a recent paper [8], when the constant $c$ of (1.2) is a variable $c(t)$, we generalized the results of [1] and obtained the following results. If $|c(t)| \neq 1$, then the operator $A$ has continuous inverse $A^{-1}$ on $C_{T}$, satisfying

(1)

$$
\left[A^{-1} f\right](t)= \begin{cases}f(t)+\sum_{j=1}^{\infty} \prod_{i=1}^{j} c(t-(i-1) \tau) f(t-j \tau), & c_{0}<1, \forall f \in C_{T}, \\ -\frac{f(t+\tau)}{c(t+\tau)}-\sum_{j=1}^{\infty} \prod_{i=1}^{j+1} \frac{1}{c(t+i \tau)} f(t+j \tau+\tau), & \sigma>1, \forall f \in C_{T} .\end{cases}
$$

(2)

$$
\int_{0}^{T}\left|\left[A^{-1} f\right](t)\right| d t \leq \begin{cases}\frac{1}{1-c_{0}} \int_{0}^{T}|f(t)| d t, & c_{0}<1, \forall f \in C_{T} \\ \frac{1}{\sigma-1} \int_{0}^{T}|f(t)| d t, & \sigma>1, \forall f \in C_{T}\end{cases}
$$

Using the above results, we have obtained some existence results of periodic solutions for first-order, second-order and $p$-Laplacian neutral equations with a variable parameter; see [9-11].

However, when $B$ of (1.1) is a matrix, there are few existence results of periodic solutions for neutral differential systems. In [12], when $B$ is a symmetric matrix, the authors studied a second-order $p$-Laplacian neutral functional differential system and obtained the existence of periodic solutions. In [13], when $B$ is a general matrix, the authors studied a second-order neutral differential system. But for $p$-Laplacian functional differential system, to the best our knowledge, there are no results on the existence of periodic solutions. Hence, in this paper, we will study system (1.1) and obtain the existence of periodic solutions by using the generalization of Mawhin's continuation theorem.

\section{Main lemmas}

In this section, we give some notations and lemmas which will be used in this paper. Let

$$
\begin{aligned}
& C_{T}=\left\{x \mid x \in C\left(\mathbb{R}, \mathbb{R}^{n}\right), x(t+T) \equiv x(t)\right\}, \\
& C_{T}^{1}=\left\{x \mid x \in C^{1}\left(\mathbb{R}, \mathbb{R}^{n}\right), x(t+T)=x(t)\right\},
\end{aligned}
$$

$X=C_{T}^{1}$ with the norm $\|x\|=\max \left\{|x|_{0},\left|x^{\prime}\right|_{0}\right\}, Z=C_{T}$ with the norm

$$
|x|_{0}=\max _{0 \leq t \leq T}|x(t)|, \quad|x(t)|=\left(\sum_{i=1}^{n} x_{i}^{2}\right)^{1 / 2},
$$

$U$ is a complex such that

$$
U B U^{-1}=E_{\lambda}=\operatorname{diag}\left(J_{1}, J_{2}, \ldots, J_{n}\right)
$$


is a Jordan's normal matrix, where

$$
J_{i}=\left(\begin{array}{cccccc}
\lambda_{i} & 1 & 0 & 0 & \cdots & 0 \\
0 & \lambda_{i} & 1 & 0 & \cdots & 0 \\
\cdots & \cdots & \cdots & \cdots & \cdots & \cdots \\
0 & 0 & \cdots & 0 & \lambda_{i} & 1 \\
0 & 0 & 0 & 0 & \cdots & \lambda_{i}
\end{array}\right)_{n_{i} \times n_{i}}
$$

with $\sum_{i=1}^{l} n_{i}=n,\left\{\lambda_{i}: i=1,2, \ldots, l\right\}$ is the set of eigenvalues of matrix $B$. Let

$$
A_{1}: C_{T} \rightarrow C_{T}, \quad\left[A_{1} x\right](t)=x(t)-B x(t-\tau) .
$$

Furthermore, we suppose that $\gamma(t) \in C^{1}(\mathbb{R}, \mathbb{R})$ with $\gamma^{\prime}(t)<1, \forall t \in \mathbb{R}$. It is obvious that the function $t-\gamma(t)$ has a unique inverse denoted by $\mu(t)$.

Lemma 2.1 ([13]) Suppose that the matrix $U$ and the operator $A_{1}$ are defined by (2.1) and (2.2), respectively, and for all $i=1,2, \ldots, l,\left|\lambda_{i}\right| \neq 1$. Then $A_{1}$ has its inverse $A_{1}^{-1}: C_{T} \rightarrow C_{T}$ with the following properties:

(1) $\left\|A_{1}^{-1}\right\| \leq\left|U^{-1}\right||U| \sigma_{0}, \sigma_{0}=\sum_{i=1}^{l} \sum_{j=1}^{n_{i}} \sum_{k=1}^{j} \frac{1}{\left|1-\lambda_{i}\right|^{k}}$.

(2) For all $f \in C_{T}, \int_{0}^{T}\left|\left[A_{1}^{-1} f\right](s)\right|^{p} d s \leq\left|U^{-1}\right|^{p}|U|^{p} \sigma_{1} \int_{0}^{T}|f(s)|^{p} d s, p \in[1,+\infty)$, where

$$
\sigma_{1}= \begin{cases}\sum_{i=1}^{l} \sum_{j=1}^{n_{i}}\left(\sum_{k=1}^{j} \frac{1}{\left|1-\lambda_{i}\right|^{k}}\right)^{2}, & p=2, \\ n^{\frac{2-p}{2}}\left[\sum_{i=1}^{l} \sum_{j=1}^{n_{i}}\left(\sum_{k=1}^{j} \frac{1}{\left|1-\lambda_{i}\right|^{k}}\right)^{q}\right]^{\frac{p}{q}}, & p \in[1,2), \\ {\left[\sum_{i=1}^{l} \sum_{j=1}^{n_{i}}\left(\sum_{k=1}^{j} \frac{1}{\left|1-\lambda_{i}\right|^{k}}\right)^{q}\right]^{\frac{p}{q}},} & p \in[2,+\infty)\end{cases}
$$

and $q>0$ is a constant with $1 / p+1 / q=1$.

(3) $A_{1}^{-1} f \in C_{T}^{1},\left[A_{1}^{-1} f\right]^{\prime}(t)=\left[A_{1}^{-1} f^{\prime}\right](t)$, for all $f \in C_{T}^{1}, t \in \mathbb{R}$.

Definition 2.1 ([14]) Let $X$ and $Z$ be two Banach spaces with norms $\|\cdot\|_{X},\|\cdot\|_{Z}$, respectively. A continuous operator

$$
M: X \cap \operatorname{dom} M \rightarrow Z
$$

is said to be quasi-linear if

(i) $\operatorname{Im} M:=M(X \cap \operatorname{dom} M)$ is a closed subset of $Z$;

(ii) $\operatorname{Ker} M:=\{x \in X \cap \operatorname{dom} M: M x=0\}$ is linearly homeomorphic to $\mathbb{R}^{n}, n<\infty$.

Definition 2.2 ([14]) Let $\Omega \subset X$ be an open and bounded set with the origin $\theta \in \Omega, N_{\lambda}$ : $\bar{\Omega} \rightarrow Z, \lambda \in[0,1]$ is said to be $M$-compact in $\bar{\Omega}$ if there exists a subset $Z_{1}$ of $Z$ satisfying $\operatorname{dim} Z_{1}=\operatorname{dim} \operatorname{Ker} M$ and an operator $R: \bar{\Omega} \times[0,1] \rightarrow X_{2}$ being continuous and compact such that for $\lambda \in[0,1]$,

(a) $(I-Q) N_{\lambda}(\bar{\Omega}) \subset \operatorname{Im} M \subset(I-Q) Z$,

(b) $Q N_{\lambda} x=0, \lambda \in(0,1) \Leftrightarrow Q N x=0, \forall x \in \Omega$,

(c) $R(\cdot, 0) \equiv 0$ and $\left.R(\cdot, \lambda)\right|_{\sum_{\lambda}}=\left.(I-P)\right|_{\sum_{\lambda}}$,

(d) $M[P+R(\cdot, \lambda)]=(I-Q) N_{\lambda}, \lambda \in[0,1]$ 
where $X_{2}$ is the complement space of $\operatorname{Ker} M$ in $\mathrm{X}$, i.e., $X=\operatorname{Ker} M \oplus X_{2} ; P, Q$ are two projectors satisfying $\operatorname{Im} P=\operatorname{Ker} M, \operatorname{Im} Q=Z_{1}, N=N_{1}, \sum_{\lambda}=\left\{x \in \bar{\Omega}: M x=N_{\lambda} x\right\}$.

Lemma 2.2 ([14]) Let $X$ and $Z$ be two Banach spaces with norms $\|\cdot\|_{X},\|\cdot\|_{Z}$, respectively and $\Omega \subset X$ be an open and bounded nonempty set. Suppose

$$
M: X \cap \operatorname{dom} M \rightarrow Z
$$

is quasi-linear and $N_{\lambda}: \bar{\Omega} \rightarrow Z, \lambda \in[0,1]$ is $M$-compact in $\bar{\Omega}$. In addition, if the following conditions hold:

$\left(\mathrm{A}_{1}\right) \quad M x \neq N_{\lambda} x, \forall(x, \lambda) \in \partial \Omega \times(0,1) ;$

$\left(\mathrm{A}_{2}\right) Q N x \neq 0, \forall x \in \operatorname{Ker} M \cap \partial \Omega$;

$\left(\mathrm{A}_{3}\right) \operatorname{deg}\{\mathrm{Q} N, \Omega \cap \operatorname{Ker} M, 0\} \neq 0, J: \operatorname{Im} Q \rightarrow \operatorname{Ker} M$ is a homeomorphism.

Then the abstract equation $M x=N x$ has at least one solution in $\operatorname{dom} M \cap \bar{\Omega}$.

Lemma $2.3([15])$ Let $s, \sigma \in C(\mathbb{R}, \mathbb{R})$ with $s(t+T) \equiv s(t)$ and $\sigma(t+T) \equiv \sigma(t)$. Suppose that the function $t-\sigma(t)$ has a unique inverse $\mu(t), \forall t \in \mathbb{R}$. Then $s(\mu(t+T)) \equiv s(\mu(t))$.

For fixed $l \in Z$ and $a \in \mathbb{R}^{n}$, define

$$
G_{l}(a)=\frac{1}{T} \int_{0}^{T} \varphi_{p}^{-1}(a+l(t)) d t
$$

Lemma 2.4 ([16]) The function $G_{l}$ has the following properties:

(1) For any fixed $l \in Z$, there must be a unique $\tilde{a}=\tilde{a}(l)$ such that the equation

$$
G_{l}(a)=0 .
$$

(2) The function a $: Z \rightarrow \mathbb{R}^{n}$ defined as above is continuous and sends bounded sets into bounded sets.

Lemma 2.5 ([17]) Let $p \in(1,+\infty)$ be a constant, $s \in C(\mathbb{R}, \mathbb{R})$ such that $s(t) \equiv s(t+T)$, $u \in X$. Then

$$
\int_{0}^{T}|u(t)-u(t-s(t))|^{p} d t \leq 2\left(\max _{t \in[0, T]}|s(t)|\right)^{p} \int_{0}^{T}\left|u^{\prime}(t)\right|^{p} d t .
$$

\section{Main results}

For convenience of applying Lemma 2.2, the operators $A, M, N_{\lambda}$ are defined by

$$
\begin{aligned}
& A: Z \rightarrow Z, \quad(A x)(t)=x(t)-B x(t-\tau), \quad t \in \mathbb{R}, \\
& M: \operatorname{dom} M \cap X \rightarrow Z, \quad(M x)(t)=\left(\varphi_{p}\left[(A x)^{\prime}\right]\right)^{\prime}(t), \quad t \in \mathbb{R}, \\
& N_{\lambda}: Z \rightarrow Z, \quad\left(N_{\lambda} x\right)(t)=-\lambda f(x(t)) x^{\prime}(t)-\lambda g(x(t-\gamma(t)))+\lambda e(t), \quad t \in \mathbb{R}, \lambda \in[0,1],
\end{aligned}
$$


where $\operatorname{dom} M=\left\{x \in X: \varphi_{p}\left[(A x)^{\prime}\right] \in C_{T}^{1}\right\}$. For convenience of the proof, let

$$
F(t, x)=-f(x(t)) x^{\prime}(t)-g(x(t-\gamma(t)))+e(t),
$$

then $\left(N_{\lambda} x\right)(t)=\lambda F$. By (3.1)-(3.3), Eq. (1.1) is equivalent to the operator equation $N x=M x$, where $N_{1}=N$. Then we have

$$
\begin{aligned}
& \operatorname{Ker} M=\left\{x \in \operatorname{dom} M \cap X: x(t)=a, a \in \mathbb{R}^{n}, t \in \mathbb{R}\right\}, \\
& \operatorname{Im} M=\left\{z \in Z: \int_{0}^{T} z(s) d s=\theta\right\} .
\end{aligned}
$$

Since $\operatorname{Ker} M \cong \mathbb{R}^{n}, \operatorname{Im} M$ is a closed set in $Z$, then we have the following.

Lemma 3.1 Let $M$ be as defined by (3.2), then $M$ is a quasi-linear operator.

Let

$$
\begin{aligned}
& P: X \rightarrow \operatorname{Ker} M, \quad(P x)(t)=x(0), \quad t \in \mathbb{R}, \\
& Q: Z \rightarrow Z / \operatorname{Im} M, \quad(Q z)(t)=\frac{1}{T} \int_{0}^{T} z(s) d s, \quad t \in \mathbb{R} .
\end{aligned}
$$

Lemma 3.2 Iff, $g, e, \gamma$ satisfy the above conditions, then $N_{\lambda}$ is M-compact.

Proof Let $Z_{1}=\operatorname{Im} Q$. For any bounded set $\bar{\Omega} \subset X \neq \emptyset$, define $R: \bar{\Omega} \times[0,1] \rightarrow \operatorname{Ker} P$,

$$
R(x, \lambda)(t)=A^{-1}\left\{\int_{0}^{t} \varphi_{q}\left[a_{x}+\int_{0}^{s} \lambda(F(r, x(r))-(Q F)(r)) d r\right] d s\right\}, \quad t \in[0, T]
$$

where $F$ is defined by (3.4) and $a_{x}$ is a constant vector in $\mathbb{R}^{n}$ which depends on $x$. By Lemma 2.4, we know that $a_{x}$ exists uniquely. Hence, $R(x, \lambda)(t)$ is well defined.

We first show that $R(\cdot, \lambda)$ is completely continuous on $\bar{\Omega} \times[0,1]$. Let

$$
G_{\lambda}(t)=\int_{0}^{t} \varphi_{q}\left[a_{x}+\int_{0}^{s} \lambda(F(r, x(r))-(Q F)(r)) d r\right] d s, \quad t \in[0, T],
$$

we have

$$
R(x, \lambda)(t)=\left[A^{-1} G_{\lambda}\right](t) .
$$

From the properties of $f, g, e, \gamma$, obviously, $\forall x \in \bar{\Omega}, G_{\lambda}(t) \in C_{T}$. Then by Lemma $2.1 R(x, \lambda)$ is uniformly bounded on $\bar{\Omega} \times[0,1]$. Now, we show $R(x, \lambda)$ is equicontinuous. $\forall t_{1}, t_{2} \in[0, T]$, $\varepsilon>0$ is sufficiently small, then there exists $\delta>0$, for $\left|t_{1}-t_{2}\right|<\delta$, by $G_{\lambda}, A^{-1} G_{\lambda} \in C_{T}$ we have

$$
\left|\left[A^{-1} G_{\lambda}\right]\left(t_{1}\right)-\left[A^{-1} G_{\lambda}\right]\left(t_{2}\right)\right|<\varepsilon .
$$

Hence, $R(x, \lambda)$ is equicontinuous on $\bar{\Omega} \times[0,1]$. By using the Arzelà-Ascoli theorem, we have $R(x, \lambda)$ is completely continuous on $\bar{\Omega} \times[0,1]$. 
Yang and Du Journal of Inequalities and Applications 2012, 2012:270

Page 6 of 13

http://www.journalofinequalitiesandapplications.com/content/2012/1/270

Secondly, we show that $N_{\lambda}$ is $M$-compact in four steps, ie., the conditions of Defineion 2.2 are all satisfied.

Step 1. By $Q^{2}=Q$, we have $Q(I-Q) N_{\lambda}(\bar{\Omega})=\theta$, so $(I-Q) N_{\lambda}(\bar{\Omega}) \subset \operatorname{Ker} Q=\operatorname{Im} M$, here $\theta$ is an $n$-dimension zero vector. On the other hand, $\forall z \in \operatorname{Im} M$. Clearly, $Q z=\theta$, so $z=z-Q z=$ $(I-Q) z$, then $z \in(I-Q) Z$. So, we have

$$
(I-Q) N_{\lambda}(\bar{\Omega}) \subset \operatorname{Im} M \subset(I-Q) Z
$$

Step 2. We show that $Q N_{\lambda} x=\theta, \lambda \in(0,1) \Leftrightarrow Q N x=\theta, \forall x \in \Omega$. Because $Q N_{\lambda} x=$ $\frac{1}{T} \int \lambda F d r=\theta$, we get $\frac{1}{T} \int F d r=\theta$, ie., $Q N x=\theta$. The inverse is true.

Step 3. When $\lambda=0$, from the above proof, we have $a_{x}=\theta$. So, we get $R(\cdot, 0)=\theta . \forall x \in$ $\sum_{\lambda}=\left\{x \in \bar{\Omega}: M x=N_{\lambda} x\right\}$, we have $\left(\varphi_{p}\left[(A x)^{\prime}\right]\right)^{\prime}=\lambda F$ and $Q F=\theta$. In this case, when $a_{x}=$ $\varphi_{p}\left[(A x)^{\prime}(0)\right]$, we have

$$
\begin{aligned}
G_{\lambda}(T) & =\int_{0}^{T} \varphi_{q}\left[a_{x}+\int_{0}^{s} \lambda(F(r, x(r))-(Q F)(r)) d r\right] d s \\
& =\int_{0}^{T} \varphi_{q}\left[\varphi_{p}\left[(A x)^{\prime}(0)\right]+\int_{0}^{s} \lambda F(r, x(r)) d r\right] d s \\
& =\int_{0}^{T} \varphi_{q}\left[\varphi_{p}\left[(A x)^{\prime}(0)\right]+\int_{0}^{s}\left(\varphi_{p}\left[(A x)^{\prime}(r)\right]\right)^{\prime} d r\right] d s \\
& =\int_{0}^{T}(A x)^{\prime}(s) d s \\
& =(A x)(T)-(A x)(0)=\theta .
\end{aligned}
$$

Hence,

$$
\begin{aligned}
R(x, \lambda)(t) & =A^{-1}\left\{\int_{0}^{t} \varphi_{q}\left[\varphi_{p}\left[(A x)^{\prime}(0)\right]+\int_{0}^{s} \lambda(F(r, x(r))-(Q F)(r)) d r\right] d s\right\} \\
& =A^{-1}\left\{\int_{0}^{t} \varphi_{q}\left[\varphi_{p}\left[(A x)^{\prime}(0)\right]+\int_{0}^{s} \lambda F(r, x(r)) d r\right] d s\right\} \\
& =A^{-1}\left\{\int_{0}^{t} \varphi_{q}\left[\varphi_{p}\left[(A x)^{\prime}(0)\right]+\int_{0}^{s}\left(\varphi_{p}\left[(A x)^{\prime}(r)\right]\right)^{\prime} d r\right] d s\right\} \\
& =A^{-1}\left\{\int_{0}^{t}(A x)^{\prime}(s) d s\right\} \\
& =A^{-1}[(A x)(t)-(A x)(0)] \\
& =[(I-P) x](t) .
\end{aligned}
$$

Step 4. $\forall x \in \bar{\Omega}$, we have

$$
\begin{aligned}
M & {[P x+R(x, \lambda)](t) } \\
& =\left(\varphi_{p}\left(\left[(A x)(0)+A A^{-1}\left\{\int_{0}^{t} \varphi_{q}\left[a_{x}+\int_{0}^{s} \lambda(F(r, x(r))-(Q F)(r)) d r\right] d s\right\}\right]^{\prime}\right)\right)^{\prime} \\
& =\left(\varphi_{p}\left(\left\{\int_{0}^{t} \varphi_{q}\left[a_{x}+\int_{0}^{s} \lambda(F(r, x(r))-(Q F)(r)) d r\right] d s\right\}\right)\right)^{\prime}
\end{aligned}
$$




$$
\begin{aligned}
& =\left(\varphi_{p}\left(\varphi_{q}\left[a_{x}+\int_{0}^{t} \lambda(F(r, x(r))-(Q F)(r)) d r\right]\right)\right)^{\prime} \\
& =\left(a_{x}+\int_{0}^{t} \lambda(F(r, x(r))-(Q F)(r)) d r\right)^{\prime} \\
& =\left[(I-Q) N_{\lambda} x\right](t) .
\end{aligned}
$$

Hence, $N_{\lambda}$ is $M$-compact in $\bar{\Omega}$.

Theorem 3.3 Suppose that $\int_{0}^{T} e(s) d s=\theta, \lambda_{1}, \lambda_{2}, \ldots, \lambda_{l}$ are eigenvalues of the matrix $B$ with $\left|\lambda_{i}\right| \neq 1, i=1,2, \ldots l$, and there exist positive constants $D>0, l>0$ and $\sigma>0$ such that

$\left(\mathrm{H}_{1}\right) x_{i} g_{i}\left(x_{i}\right)>0, \forall x_{i} \in \mathbb{R},\left|x_{i}\right|>D$, for each $i=1,2, \ldots, n$,

$\left(\mathrm{H}_{2}\right)\left|g_{i}\left(u_{1}\right)-g_{i}\left(u_{2}\right)\right| \leq l\left|u_{1}-u_{2}\right|, u_{1}, u_{2} \in \mathbb{R}$, for each $i=1,2, \ldots, n$,

$\left(\mathrm{H}_{3}\right)\left|f_{i}\left(x_{i}\right)\right| \geq \sigma, x_{i} \in \mathbb{R}$, for each $i=1,2, \ldots, n$.

Then Eq. (1.1) has at least one T-periodic solution if one of the following two conditions is satisfied:

$$
\begin{aligned}
& \sigma>\sqrt{2} l \max _{t \in[0, T]}|\gamma(t)| \quad \text { for } 1<q<2 \text { or } \\
& \sigma>\sqrt{2} l \max _{t \in[0, T]}|\gamma(t)|, \quad\left|U^{-1}\right||U| \sigma_{0} \sqrt{n} T f_{R_{2}}<1 \quad \text { for } q=2,
\end{aligned}
$$

where $f_{R_{2}}=\max _{|x| \leq R_{2}}|f(x)|, R_{2}$ is defined by (3.14).

Proof We complete the proof in three steps.

Step 1. Let $\Omega_{1}=\left\{x \in \operatorname{dom} M: M x=N_{\lambda} x, \lambda \in(0,1)\right\}$. We show that $\Omega_{1}$ is a bounded set. If $x \in \Omega_{1}$, then $M x=N_{\lambda} x$, i.e.,

$$
\left(\varphi_{p}\left[(A x)^{\prime}\right]\right)^{\prime}=-\lambda f(x(t)) x^{\prime}(t)-\lambda g(x(t-\gamma(t)))+\lambda e(t) .
$$

Integrating both sides of (3.5) over [0, $T]$, we have

$$
\int_{0}^{T} g(x(t-\gamma(t))) d t=\theta
$$

which together with assumption $\left(\mathrm{H}_{1}\right)$ leads to the fact that there exists a point $\xi_{i} \in \mathbb{R}$ such that

$$
\left|x_{i}\left(\xi_{i}-\gamma\left(\xi_{i}\right)\right)\right| \leq D, \quad \text { for each } i=1,2, \ldots, n
$$

Let $\xi_{i}-\gamma\left(\xi_{i}\right)=k T+\eta_{i}, k \in \mathbb{Z}, \eta_{i} \in[0, T]$. Then

$$
\left|x_{i}\left(\eta_{i}\right)\right| \leq D, \quad \text { for each } i=1,2, \ldots, n
$$

Thus,

$$
\left|x_{i}\right| \leq D+\int_{0}^{T}\left|x_{i}^{\prime}(s)\right| d s, \quad \text { for each } i=1,2, \ldots, n
$$


By (3.6), we have

$$
\begin{aligned}
|x| & =\left(x_{1}^{2}+x_{2}^{2}+\cdots+x_{n}^{2}\right)^{\frac{1}{2}} \\
& \leq \sqrt{n}\left(D+\int_{0}^{T}\left|x^{\prime}(s)\right| d s\right)
\end{aligned}
$$

and

$$
|x|_{0} \leq \sqrt{n}\left(D+\int_{0}^{T}\left|x^{\prime}(s)\right| d s\right)
$$

On the other hand, multiplying the two sides of Eq. (3.5) by $\left[x^{\prime}(t)\right]^{\top}$ from the left side and integrating them over $[0, T]$, we have

$$
\begin{aligned}
\int_{0}^{T}\left[x^{\prime}(t)\right]^{\top}\left(\varphi_{p}\left[(A x)^{\prime}\right]\right)^{\prime} d t= & -\lambda \int_{0}^{T}\left[x^{\prime}(t)\right]^{\top} f(x(t)) x^{\prime}(t) d t \\
& -\lambda \int_{0}^{T}\left[x^{\prime}(t)\right]^{\top} g(x(t-\gamma(t))) d t \\
& +\lambda \int_{0}^{T}\left[x^{\prime}(t)\right]^{\top} e(t) d t .
\end{aligned}
$$

Let $\omega(t)=\varphi_{p}\left[(A x)^{\prime}(t)\right]$, then

$$
\int_{0}^{T}\left[x^{\prime}(t)\right]^{\top}\left(\varphi_{p}\left[(A x)^{\prime}\right]\right)^{\prime} d t=\int_{0}^{T}\left\{A^{-1}\left(\varphi_{q}(\omega(t))\right)\right\}^{\top} d \omega(t)=0 .
$$

By (3.9), we have

$$
\begin{aligned}
& \left|\sum_{i=1}^{n} \int_{0}^{T} f_{i}\left(x_{i}(t)\right)\left[x_{i}^{\prime}(t)\right]^{2} d t\right| \\
& \quad \leq\left|\int_{0}^{T}\left[x^{\prime}(t)\right]^{\top} g(x(t-\gamma(t))) d t\right|+\left|\int_{0}^{T}\left[x^{\prime}(t)\right]^{\top} e(t) d t\right| .
\end{aligned}
$$

By assumption $\left(\mathrm{H}_{3}\right)$, we have

$$
\begin{aligned}
\sigma \sum_{i=1}^{n} \int_{0}^{T}\left|x_{i}^{\prime}(t)\right|^{2} d t & \leq \sum_{i=1}^{n} \int_{0}^{T}\left|f_{i}\left(x_{i}(t)\right)\right|\left[x_{i}^{\prime}(t)\right]^{2} d t \\
& =\left|\sum_{i=1}^{n} \int_{0}^{T} f_{i}\left(x_{i}(t)\right)\left[x_{i}^{\prime}(t)\right]^{2} d t\right| .
\end{aligned}
$$

From (3.10) and (3.11), we have

$$
\sigma \sum_{i=1}^{n} \int_{0}^{T}\left|x_{i}^{\prime}(t)\right|^{2} d t \leq \int_{0}^{T}\left|\left[x^{\prime}(t)\right]^{\top} g(x(t-\gamma(t)))\right| d t+\int_{0}^{T}\left|\left[x^{\prime}(t)\right]^{\top} e(t)\right| d t .
$$


From $\int_{0}^{T}\left[x^{\prime}(t)\right]^{\top} g(x(t)) d t=0$, assumption $\left(\mathrm{H}_{2}\right)$, Lemma 2.5 and (3.12), we have

$$
\begin{aligned}
\sigma & \sum_{i=1}^{n} \int_{0}^{T}\left|x_{i}^{\prime}(t)\right|^{2} d t \\
\leq & \int_{0}^{T}\left|\left[x^{\prime}(t)\right]^{T}[g(x(t))-g(x(t-\gamma(t)))]\right| d t+\int_{0}^{T}\left|\left[x^{\prime}(t)\right]^{T} e(t)\right| d t \\
\leq & \sum_{i=1}^{n} l \int_{0}^{T}\left|x_{i}^{\prime}(t)\right|\left|x_{i}(t)-x_{i}(t-\gamma(t))\right| d t+\left(\int_{0}^{T}\left|x^{\prime}(t)\right|^{2} d t\right)^{1 / 2}\left(\int_{0}^{T}|e(t)|^{2} d t\right)^{1 / 2} \\
\leq & \sum_{i=1}^{n} l\left(\int_{0}^{T}\left|x_{i}^{\prime}(t)\right|^{2} d t\right)^{1 / 2}\left(\int_{0}^{T}\left|x_{i}(t)-x_{i}(t-\gamma(t))\right|^{2} d t\right)^{1 / 2} \\
& +\left(\int_{0}^{T}\left|x^{\prime}(t)\right|^{2} d t\right)^{1 / 2}\left(\int_{0}^{T}|e(t)|^{2} d t\right)^{1 / 2} \\
\leq & \sum_{i=1}^{n} \sqrt{2} l \max _{t \in[0, T]}|\gamma(t)| \int_{0}^{T}\left|x_{i}^{\prime}(t)\right|^{2} d t+\left(\int_{0}^{T}\left|x^{\prime}(t)\right|^{2} d t\right)^{1 / 2}\left(\int_{0}^{T}|e(t)|^{2} d t\right)^{1 / 2} .
\end{aligned}
$$

Since $\sigma>\sqrt{2} l \max _{t \in[0, T]}|\gamma(t)|$, by (3.13), there exists a positive constant $R_{1}$ such that

$$
\int_{0}^{T}\left|x^{\prime}(s)\right| d s \leq R_{1}
$$

Then by (3.8),

$$
|x|_{0} \leq \sqrt{n}\left(D+R_{1}\right):=R_{2}
$$

By (3.5), we have

$$
\left|\left(\varphi_{p}\left[(A x)^{\prime}\right]\right)^{\prime}\right| \leq f_{R_{2}}\left|x^{\prime}(t)\right|+g_{R_{2}}+|e|_{0},
$$

where $f_{R_{2}}=\max _{|x| \leq R_{2}}|f(x)|, g_{R_{2}}=\max _{|x| \leq R_{2}}|g(x)|$. Take $\varphi_{p}\left[(A x)^{\prime}(t)\right]=y(t)$, then

$$
\left|y^{\prime}\right|_{0} \leq f_{R_{2}}\left|x^{\prime}(t)\right|+g_{R_{2}}+|e|_{0}
$$

and $(A x)^{\prime}(t)=\varphi_{q}(y(t))$. Because there exists a $t_{i} \in[0, T]$ such that $y\left(t_{i}\right)=0, i=1,2, \ldots, n$, so by (3.15), we get

$$
|y(t)| \leq \sqrt{n} T\left|y^{\prime}\right|_{0} \leq \sqrt{n} T f_{R_{2}}\left|x^{\prime}(t)\right|+\sqrt{n} T g_{R_{2}}+\sqrt{n} T|e|_{0}
$$

and

$$
\left|(A x)^{\prime}(t)\right| \leq\left(\sqrt{n} T f_{R_{2}}\left|x^{\prime}(t)\right|+\sqrt{n} T g_{R_{2}}+\sqrt{n} T|e|_{0}\right)^{q-1} .
$$

By (3.16) and Lemma 2.1, we have

$$
\begin{aligned}
\left|x^{\prime}(t)\right| & =\left|\left[A^{-1} A x^{\prime}\right](t)\right| \leq\left|U^{-1}\right||U| \sigma_{0}\left|(A x)^{\prime}(t)\right| \\
& \leq\left|U^{-1}\right||U| \sigma_{0}\left(\sqrt{n} T f_{R_{2}}\left|x^{\prime}(t)\right|+\sqrt{n} T g_{R_{2}}+\sqrt{n} T|e|_{0}\right)^{q-1} .
\end{aligned}
$$


Now, we consider $\left(\sqrt{n} T f_{R_{2}}\left|x^{\prime}(t)\right|+\sqrt{n} T g_{R_{2}}+\sqrt{n} T|e|_{0}\right)^{q-1}$. In the formal case, we get

$$
\begin{aligned}
& \left(\sqrt{n} T f_{R_{2}}\left|x^{\prime}(t)\right|+\sqrt{n} T g_{R_{2}}+\sqrt{n} T|e|_{0}\right)^{q-1} \\
& \quad=\left(\sqrt{n} T f_{R_{2}}\left|x^{\prime}(t)\right|\right)^{q-1}\left(1+\frac{\sqrt{n} T g_{R_{2}}+\sqrt{n} T|e|_{0}}{\sqrt{n} T f_{R_{2}}\left|x^{\prime}(t)\right|}\right)^{q-1} .
\end{aligned}
$$

By classical elementary inequalities, we see that there is a constant $h(p)>0$, which is dependent on $p$ only, such that

$$
(1+x)^{p}<1+(1+p) x, \quad \forall x \in(0, h(p)] .
$$

Case 2.1. If $\frac{\sqrt{n} T g_{R_{2}}+\sqrt{n} T|e|_{0}}{\sqrt{n} T f_{R_{2}}\left|x^{\prime}(t)\right|}>h$, then

$$
\left|x^{\prime}(t)\right|<\frac{\sqrt{n} T g_{R_{2}}+\sqrt{n} T|e|_{0}}{\sqrt{n} T f_{R_{2}} h}:=M_{1} .
$$

Case 2.2. If $\frac{\sqrt{n} T g_{R_{2}}+\sqrt{n} T|e|_{0}}{\sqrt{n} T f_{R_{2}}\left|x^{\prime}(t)\right|} \leq h$, by (3.18) and (3.19), we have

$$
\begin{aligned}
& \left(\sqrt{n} T f_{R_{2}}\left|x^{\prime}(t)\right|+\sqrt{n} T g_{R_{2}}+\sqrt{n} T|e|_{0}\right)^{q-1} \\
& \quad=\left(\sqrt{n} T f_{R_{2}}\left|x^{\prime}(t)\right|\right)^{q-1}\left(1+\frac{\sqrt{n} T g_{R_{2}}+T|e|_{0}}{\sqrt{n} T f_{R_{2}}\left|x^{\prime}(t)\right|}\right)^{q-1} \\
& \leq\left(\sqrt{n} T f_{R_{2}}\left|x^{\prime}(t)\right|\right)^{q-1}\left(1+\frac{q\left(\sqrt{n} T g_{R_{2}}+\sqrt{n} T|e|_{0}\right)}{\sqrt{n} T f_{R_{2}}\left|x^{\prime}(t)\right|}\right) \\
& \quad=\left(\sqrt{n} T f_{R_{2}}\right)^{q-1}\left|x^{\prime}(t)\right|^{q-1}+q\left(\sqrt{n} T g_{R_{2}}+\sqrt{n} T|e|_{0}\right)\left(\sqrt{n} T f_{R_{2}}\right)^{q-2}\left|x^{\prime}(t)\right|^{q-2} .
\end{aligned}
$$

From (3.17) and (3.21), we have

$$
\begin{aligned}
\left|x^{\prime}(t)\right| \leq & \left|U^{-1}\right||U| \sigma_{0}\left(\sqrt{n} T f_{R_{2}}\right)^{q-1}\left|x^{\prime}(t)\right|^{q-1} \\
& +\left|U^{-1}\right||U| \sigma_{0} q\left(\sqrt{n} T g_{R_{2}}+T|e|_{0}\right)\left(\sqrt{n} T f_{R_{2}}\right)^{q-2}\left|x^{\prime}(t)\right|^{q-2} .
\end{aligned}
$$

When $q=2$, from $\left|U^{-1}\right||U| \sigma_{0} \sqrt{n} T f_{R_{2}}<1$, we know that there exists a constant $M_{2}>0$ such that

$$
\left|x^{\prime}(t)\right| \leq M_{2}
$$

When $1<q<2$, there must be a constant $M_{3}>0$ such that

$$
\left|x^{\prime}(t)\right| \leq M_{3} .
$$

Hence, from (3.14), (3.20), (3.23) and (3.24), we have

$$
\|x\|<\max \left\{R_{2}, M_{1}, M_{2}, M_{3}\right\}+1:=L .
$$


Step 2. Let $\Omega_{2}=\{x \in \operatorname{Ker} M: Q N x=\theta\}$, we shall prove that $\Omega_{2}$ is a bounded set. $\forall x \in \Omega_{2}$, then $x=a, a \in \mathbb{R}^{n}$, we have $g_{i}\left(a_{i}\right)=0$ for each $i=1,2, \ldots, n$. By assumption $\left(\mathrm{H}_{1}\right)$ we have $\left|a_{i}\right| \leq D$ and $|a| \leq \sqrt{n} D$. So, $\Omega_{2}$ is a bounded set.

Step 3. Let $\Omega=\{x \in X:\|x\|<L\}$, then $\Omega_{1} \cup \Omega_{2} \subset \Omega, \forall(x, \lambda) \in \partial \Omega \times(0,1)$. From the above proof, $M x \neq N_{\lambda} x$ is satisfied. Obviously, condition $\left(\mathrm{A}_{2}\right)$ of Lemma 2.2 is also satisfied. Now, we prove that condition $\left(\mathrm{A}_{3}\right)$ of Lemma 2.2 is satisfied. Take the homotopy

$$
H(x, \mu)=\mu x-(1-\mu) J Q N x, \quad x \in \bar{\Omega} \cap \operatorname{Ker} M, \mu \in[0,1],
$$

where $J: \operatorname{Im} Q \rightarrow \operatorname{Ker} M$ is a homeomorphism with $J a=a, a \in \mathbb{R}^{n} . \forall x \in \partial \Omega \cap \operatorname{Ker} M$, we have $x=a_{1} \in \mathbb{R}^{n},\left|a_{1}\right|=L>D$, then

$$
\begin{aligned}
H(x, \mu) & =a_{1} \mu-(1-\mu) \frac{1}{T} \int_{0}^{T}\left(-g\left(a_{1}\right)+e(t)\right) d t \\
& =a_{1} \mu+(1-\mu) g\left(a_{1}\right),
\end{aligned}
$$

then we have

$$
a_{1}^{\top} H(x, \mu)=a_{1}^{\top} a_{1} \mu+(1-\mu) a_{1}^{\top} g\left(a_{1}\right) .
$$

By using assumption $\left(\mathrm{H}_{1}\right)$, we have $H(x, \mu) \neq 0$. And then, by the degree theory,

$$
\begin{aligned}
\operatorname{deg}\{U Q N, \Omega \cap \operatorname{Ker} M, 0\} & =\operatorname{deg}\{H(\cdot, 0), \Omega \cap \operatorname{Ker} M, 0\} \\
& =\operatorname{deg}\{H(\cdot, 1), \Omega \cap \operatorname{Ker} M, 0\} \\
& =\operatorname{deg}\{I, \Omega \cap \operatorname{Ker} M, 0\} \neq 0 .
\end{aligned}
$$

Applying Lemma 2.2, we complete the proof.

Remark Assumption $\left(\mathrm{H}_{1}\right)$ guarantees that condition $\left(\mathrm{A}_{2}\right)$ of Lemma 2.2 is satisfied. Furthermore, using assumptions $\left(\mathrm{H}_{1}\right)-\left(\mathrm{H}_{3}\right)$, we can easily estimate prior boud of the solution to Eq. (1.1).

As an application, we consider the following example:

$$
\left(\varphi_{p}\left[(x(t)-B x(t-\pi))^{\prime}\right]\right)^{\prime}+f(x(t)) x^{\prime}(t)+g(x(t-\pi))=e(t)
$$

where

$$
x(t)=\left(\begin{array}{l}
x_{1}(t) \\
x_{2}(t)
\end{array}\right) \in \mathbb{R}^{3}, \quad g(x)=\left(\begin{array}{c}
\frac{1}{100} x_{1} \\
\frac{1}{100} x_{2}
\end{array}\right), \quad B=\left(\begin{array}{cc}
-1 & -3 \\
-4 & 0
\end{array}\right),
$$

$e(t)=(\sin t, \cos t)^{\top}, \tau=\gamma=\pi, p=1.5, T=2 \pi, f(x)=\left(5+\sin x_{1}, 10+\cos x_{2}\right)$.

Obviously, $\lambda_{1}=3 \neq \pm 1, \lambda_{2}=-4 \neq \pm 1$,

$$
\int_{0}^{2 \pi} e(t) d t=\left(\begin{array}{l}
\int_{0}^{2 \pi} \cos t d t \\
\int_{0}^{2 \pi} \sin t d t
\end{array}\right)=\left(\begin{array}{l}
0 \\
0
\end{array}\right) .
$$


Since

$$
x_{1} g_{1}\left(x_{1}\right)=\frac{1}{100} x_{1}^{2}>0 \quad \text { for }\left|x_{1}\right|>D>0, \quad x_{2} g_{2}\left(x_{2}\right)=\frac{1}{100} x_{2}^{2}>0 \quad \text { for }\left|x_{2}\right|>D>0
$$

so assumption $\left(\mathrm{H}_{1}\right)$ is satisfied. Take $l=\frac{1}{100}$, then

$$
\left|g_{i}\left(u_{1}\right)-g_{i}\left(u_{2}\right)\right| \leq \frac{1}{100}\left|u_{1}-u_{2}\right|, \quad u_{1}, u_{2} \in \mathbb{R}, \text { for each } i=1,2
$$

and assumption $\left(\mathrm{H}_{2}\right)$ is satisfied. Take $\sigma=4$, then

$$
\left|f_{1}\left(x_{1}\right)\right|=\left|5+\sin x_{1}\right| \geq 4, \quad\left|f_{2}\left(x_{2}\right)\right|=\left|10+\cos x_{2}\right| \geq 4,
$$

and assumption $\left(\mathrm{H}_{3}\right)$ is satisfied. Hence, assumptions $\left(\mathrm{H}_{1}\right)-\left(\mathrm{H}_{3}\right)$ are all satisfied. Take

$$
U=\left(\begin{array}{cc}
-1 & 1 \\
4 & 3
\end{array}\right), \quad U^{-1}=\left(\begin{array}{cc}
-\frac{3}{7} & \frac{1}{7} \\
\frac{4}{7} & \frac{1}{7}
\end{array}\right)
$$

such that

$$
U B U^{-1}=\left(\begin{array}{cc}
3 & 0 \\
0 & -4
\end{array}\right) .
$$

Take $\gamma(t)=\pi$, then

$$
\sigma>\sqrt{2} l \max _{t \in[0, T]}|\gamma(t)|
$$

By using Theorem 3.3, we know that Eq. (3.25) has at least one $2 \pi$-periodic solution.

\section{Competing interests}

The authors declare that they have no competing interests.

\section{Authors' contributions}

The first author QY gave an example for verifying the paper's results. The corresponding author BD gave the proof for all the theorems. QY and BD read and approved the final manuscript.

\section{Acknowledgements}

This work was supported by NSF of Jiangsu education office (11KJB110002), Postdoctoral Fundation of Jiangsu (1102096C), Postdoctoral Fundation of China (2012M511296) and Jiangsu province fund (BK2011407).

Received: 24 April 2012 Accepted: 8 November 2012 Published: 27 November 2012

\section{References}

1. Zhang, M: Periodic solutions of linear and quasilinear neutral functional differential equations. J. Math. Anal. Appl. 189, 378-392 (1995)

2. Lu, S, Ge, W, Zheng, Z: Periodic solutions to neutral differential equation with deviating arguments. Appl. Math. Comput. 152, 17-27 (2004)

3. Kaufmann, ER, Raffoul, YN: Periodic solutions for a neutral nonlinear dynamical equation on a time scale. J. Math. Anal. Appl. 319, 315-325 (2006)

4. Li, Y: Periodic solutions for delay Lotka-Volterra competition systems. J. Math. Anal. Appl. 246, 230-244 (2000)

5. Fan, M, Wang, K: Global periodic solutions of a generalized n-species Gilpin-Ayala competition model. Comput. Math. Appl. 40, 1141-1151 (2000)

6. Chen, F, Lin, F, Chen, X: Sufficient conditions for the existence of positive periodic solutions of a class of neutral delay models with feedback control. Appl. Math. Comput. 158, 45-68 (2004)

7. Lu, S, Ge, W: On the existence of periodic solutions for neutral functional differential equation. Nonlinear Anal. TMA 54, 1285-1306 (2003) 
8. Du, B, Guo, L, Ge, W, Lu, S: Periodic solutions for generalized Liénard neutral equation with variable parameter. Nonlinear Anal. TMA 70, 2387-2394 (2008)

9. Du, B, Wang, X: Periodic solutions for a second-order neutral differential equation with variable parameter and multiple deviating arguments. Electron. J. Differ. Equ. 2010, 1-10 (2010)

10. Du, B, Zhao, J, Ge, W: Periodic solutions for a neutral differential equation with variable parameter. Topol. Methods Nonlinear Anal. 33, 275-283 (2009)

11. Du, B, Sun, B: Periodic solutions to a $p$-Laplacian neutral Duffing equation with variable parameter. Electron. J. Qual. Theory Differ. Equ. 2011, 55 (2011)

12. Lu, S: Periodic solutions to a second order $p$-Laplacian neutral functional differential system. Nonlinear Anal. TMA 69, 4215-4229 (2008)

13. $L u, S, X u, Y, X i a, D$ : New properties of the D-operator and its applications on the problem of periodic solutions to neutral functional differential system. Nonlinear Anal. TMA 74, 3011-3021 (2011)

14. Ge, W, Ren, J: An extension of Mawhin's continuation theorem and its application to boundary value problems with a p-Laplacian. Nonlinear Anal. TMA 58, 477-488 (2004)

15. Lu, S, Ge, W: Existence of positive periodic solutions for neutral population model with multiple delays. Appl. Math. Comput. 153, 885-902 (2004)

16. Manásevich, R, Mawhin, J: Periodic solutions for nonlinear systems with p-Laplacian-like operators. J. Differ. Equ. 145, 367-393 (1998)

17. Lu, S, Ge, W: Periodic solutions for a kind of Liénard equation with a deviating argument. J. Math. Anal. Appl. 289, 231-243 (2004)

doi:10.1186/1029-242X-2012-270

Cite this article as: Yang and Du: Periodic solutions to a generalized Liénard neutral functional differential system

with p-Laplacian. Journal of Inequalities and Applications 2012 2012:270.

\section{Submit your manuscript to a SpringerOpen ${ }^{\circ}$ journal and benefit from:}

- Convenient online submission

- Rigorous peer review

- Immediate publication on acceptance

- Open access: articles freely available online

- High visibility within the field

- Retaining the copyright to your article 